\title{
Stationary queue length of a single-server queue with negative arrivals and non- exponential service time distributions
}

\author{
$S K \mathrm{Koh}^{1, *}, C H \mathrm{Chin}^{1}, Y F \mathrm{Tan}^{2}, L E \mathrm{Teoh}^{1}, A H \mathrm{Pooi}^{3}$, and $Y K \mathrm{Goh}^{1}$ \\ ${ }^{1}$ Universiti Tunku Abdul Rahman, Jalan Sungai Long, Bandar Sungai Long, 43000 Kajang, Selangor, \\ Malaysia \\ ${ }^{2}$ Multimedia University, Persiaran Multimedia, 63100 Cyberjaya, Selangor, Malaysia \\ ${ }^{3}$ Sunway University, Jalan Universiti, Bandar Sunway, 47500 Subang Jaya, Selangor, Malaysia
}

\begin{abstract}
In this paper, a single-server queue with negative customers is considered. The arrival of a negative customer will remove one positive customer that is being served, if any is present. An alternative approach will be introduced to derive a set of equations which will be solved to obtain the stationary queue length distribution. We assume that the service time distribution tends to a constant asymptotic rate when time $t$ goes to infinity. This assumption will allow for finding the stationary queue length of queueing systems with non-exponential service time distributions. Numerical examples for gamma distributed service time with fractional value of shape parameter will be presented in which the steady-state distribution of queue length with such service time distributions may not be easily computed by most of the existing analytical methods.
\end{abstract}

\section{Introduction}

Queues with negative arrivals, known as G-queues, were first introduced by Gelenbe $[1,2]$ in modelling of neural networks. Since the introduction by Gelenbe, these types of queues have drawn great attention and studied extensively. The application is then widen to other areas such as communication systems, industrial engineering and manufacturing systems [3-5]. In practical applications, the term of negative customers is used to resemble viruses, inhibitory signals and orders of demand. Typically, the arrival of a negative customer to the queue will remove one positive customer according to some killing disciplines. There are two simplest forms of killing disciplines: removal of a positive customer at the head $(\mathrm{RCH})$ and removal of a positive customer at the end (RCE). Jain and Sigman [6] initiated a disaster killing strategy in which the arrival of a negative customer will remove all positive customers. Boucherie and Boxma [7] then considered the removal of a random number of positive customers upon the arrival of a negative customer. By assuming Poisson arrival of negative customers, Harrison and Pitel [8] derived expressions to find the stationary queue length and sojourn time distributions for an $\mathrm{M} / \mathrm{M} / 1$ queue. The same authors [9] then extended the model to $\mathrm{M} / \mathrm{G} / 1$ queue and expressed explicitly the stability conditions.

\footnotetext{
*Corresponding author: kohsk@utar.edu.my
} 
In this paper, we consider queueing systems with negative arrivals and RCH killing strategy. Numerical method introduced in Koh et al. [10] will be applied to find the stationary queue length distribution. The service time distribution is assumed to have constant asymptotic rate (CAR) when the time $t$ tends to infinity. With this assumption, many distributions can be taken into consideration since in reality, most of the distributions tend to constant when time $t$ goes to infinity. A set of equations for the stationary probabilities will be derived and the stationary queue length distribution can be obtained by solving the equations. Mean queue length computed by the alternative method will be compared to those obtained by the analytical method in [9] and verified with the simulation results. However, we will only find the stationary queue length distribution using the alternative method introduced in this paper since the derivation of probability generating function (pgf) to find the steady-state distribution using Laplace transform have a complex form of expression for an $\mathrm{M} / \mathrm{G} / 1$ queue.

The rest of the paper is organized as follows. Section 2 gives a description on the queueing model. The set of equations for the stationary probabilities will be derived in Section 3 and the method to solve for the numerical values will be shown in Section 4. In Section 5, expression to find the mean queue length will be derived using the analytical method in [9]. Numerical examples will be discussed in Section 6 and the concluding remarks is presented in the Section 7.

\section{Model description}

A first come first serve single server queue with negative arrivals is considered. Upon the arrival of a negative customer, one positive customer at the head will be removed if any is present. The negative customer has no effect to the system when the queue is empty. Interarrival time of both the positive and negative customers are assumed to have exponential distribution with rate $\lambda$ and $\gamma$, respectively. Service time distribution will have a constant asymptotic rate when the time $t$ tends to infinity. Examples of distributions with constant asymptotic rate are exponential, Erlang, Hyperexponential, Gamma and etc.

\section{Derivative of the stationary probabilities}

The time axis is first segmented into equal length of interval and denote $\tau_{k}$ as the interval $((k-1) \Delta t, k \Delta t]$ for $k=1,2,3, \ldots$ Let $f(t)$ be the probability density function (pdf) of the service time and

where $J$ is large enough such that $\mu_{J} \cong \lim _{k \rightarrow \infty} \mu_{k}$.

$$
\mu_{k}=\frac{f(k \Delta t)}{\int_{k \Delta t}^{\infty} f(u) d u}, \quad 1 \leq k \leq J
$$

Suppose that a positive customer arrive at time $t=0$ and receive service once enter the queue. The probability that the service is completed in the interval $\tau_{1}$ is $\mu_{1} \Delta t$. If the service is not completed in the interval $\tau_{1}, \ldots, \tau_{k-1}$, the probability that the service will be completed in $\tau_{k}$ is approximately $\mu_{k} \Delta t$ for $k=2,3,4, \ldots$ where $\mu_{k}=\mu_{J}$ for $k \geq J$.

Let $\xi_{k}, \psi_{k}$ and $\zeta_{k}$ be the states of the positive arrival, negative arrival and service processes respectively at the end of $\tau_{k}$. We define the state numbers of these processes as follows:

$$
\xi_{k}= \begin{cases}0, & \text { if there is a positive arrival in } \tau_{k} \text { with the rate } \lambda, k \geq 0 . \\ 1, & \text { if no positive arrival in } \tau_{k}, k \geq 0 .\end{cases}
$$




$$
\begin{aligned}
& \psi_{k}= \begin{cases}0, & \text { if no negative arrival in } \tau_{k}, k \geq 1 \\
1, & \text { if there is a negative arrival in } \tau_{k} \text { with the rate } \gamma, k \geq 1 .\end{cases} \\
& \zeta_{k}= \begin{cases}0, & \text { if } \quad \\
& \bullet \quad \text { the service for the positive customer ends in } \tau_{k}, \text { for } k \geq \\
& 1 ; \text { or } \\
& \text { there is a negative arrival in } \tau_{k}, k \geq 1 ; \text { or } \\
\operatorname{lin}(k, I), & \text { if the server is idle in } \tau_{k} .\end{cases}
\end{aligned}
$$

Let $n_{k}$ be the number of positive customers at the end of $\tau_{k}$. The vector that represents the number of positive customers and the states of the positive arrival, negative arrival and service processes at the end of $\tau_{k}$ is $\mathrm{Z}_{k}=\left(n_{k}, \xi_{k}, \psi_{k}, \zeta_{k}\right)$. Let $p_{n i j}^{(k)}$ be the probability that at the end of $\tau_{k}$, the number of positive customers is $n$, the states of the positive arrival, negative arrival and service processes is $i, r$ and $j$, respectively. We assume that the stationary probabilities exist where $p_{n i j}=\lim _{k \rightarrow \infty} p_{n i j}^{(k)}$.

If the system is not empty at the end of $\tau_{k-1}$ and $\mathrm{Z}_{k-1}=(n, 1,0, j)$, then one of the following events can occur in the next time interval $\tau_{k}$ :

(a) one positive customer enter the queue with the rate $\lambda$ and $Z_{k}=\left(n+1,0,0, j^{*}\right)$;

(b) one negative customer enter the queue with the rate $\gamma$ and $Z_{k}=(n-1,0,1,0)$;

(c) a completion of service with the rate $\mu_{j *}$ and $Z_{k}=(n-1,1,0,0)$;

(d) no positive or negative arrival and no completion of service which yields $\mathrm{Z}_{k}=\left(n, 1,0, j^{*}\right)$.

Suppose that the system is empty at the end of $\tau_{k-1}$ and $Z_{k-1}=(0,1,0,0)$. Then only one of the following events can occur in $\tau_{k}$ :

(e) one positive customer enter the queue with the rate $\lambda$ and $Z_{k}=(1,0,0,1)$;

(f) one negative customer enter the queue with the rate $\gamma$ and $Z_{k}=(0,0,1,0)$;

(g) no positive or negative arrival which yields $Z_{k}=(0,1,0,0)$.

For instance, if Event (e) described above occurs in $\tau_{k}$, then the probability that there is one positive customer at the end of the interval $\tau_{k}$ and $Z_{k}=(1,0,0,1)$ is given by:

$$
p_{1001}^{(k)} \cong p_{0100}^{(k-1)}(\lambda \Delta t)
$$

When $k \rightarrow \infty$, by finding the combinations of $Z_{k-1}$ and the events that could occur in $\tau_{k}$ which lead to $Z_{k}$, we will obtain the following equations.

When $n=0$ :

$$
\begin{aligned}
p_{n 100} \cong & \left(p_{n 100}+p_{n 110}\right)(1-\lambda \Delta t)(1-\gamma \Delta t)+p_{(n+1) 001}(1-\lambda \Delta t)(1-\gamma \Delta t)\left(\mu_{2} \Delta t\right) \\
+ & \sum_{j=0}^{J} p_{(n+1) 10 j}(1-\lambda \Delta t)(1-\gamma \Delta t)\left(\mu_{\min (j+1, J)} \Delta t\right)+p_{(n+1) 110}(1-\lambda \Delta t)(1-\gamma \Delta t)\left(\mu_{1} \Delta t\right) \\
p_{n 110} \cong & \cong\left(p_{n 100}+p_{n 110}+p_{(n+1) 001}\right)(1-\lambda \Delta t)(\gamma \Delta t)+p_{(n+1) 110}(1-\lambda \Delta t)(\gamma \Delta t) \\
& +\sum_{j=0}^{J} p_{(n+1) 10 j}(1-\lambda \Delta t)(\gamma \Delta t)
\end{aligned}
$$

When $n=1$ :

$$
\begin{gathered}
p_{n 102} \cong\left(p_{n 001}+p_{n 101}\right)(1-\lambda \Delta t)(1-\gamma \Delta t)\left(1-\mu_{2} \Delta t\right) \\
p_{n 10 j} \cong p_{n 10(j-1)}(1-\lambda \Delta t)(1-\gamma \Delta t)\left(1-\mu_{j} \Delta t\right), \quad \text { for } 3 \leq j \leq J-1
\end{gathered}
$$




$$
p_{n 10 J} \cong\left(p_{n 10(J-1)}+p_{n 10 J}\right)(1-\lambda \Delta t)(1-\gamma \Delta t)\left(1-\mu_{J} \Delta t\right)
$$

For $n \geq 1$ :

$$
\begin{gathered}
p_{n 001} \cong\left(p_{(n-1) 100}+p_{(n-1) 110}\right)(\lambda \Delta t) \\
p_{n 100} \cong \sum_{j=1}^{J} p_{(n+1) 00 j}(1-\lambda \Delta t)(1-\gamma \Delta t)\left(\mu_{\min (j+1, J)} \Delta t\right)+\sum_{j=0}^{J} p_{(n+1) 10 j}(1-\lambda \Delta t)(1-\gamma \Delta t)\left(\mu_{\min (j+1, J)} \Delta t\right) \\
+p_{(n+1) 110}(1-\lambda \Delta t)(1-\gamma \Delta t)\left(\mu_{1} \Delta t\right) \\
p_{n 101} \cong\left(p_{n 100}+p_{n 110}\right)(1-\lambda \Delta t)(1-\gamma \Delta t)\left(1-\mu_{1} \Delta t\right) \\
p_{n 110} \cong \sum_{j=1}^{J} p_{(n+1) 00 j}(1-\lambda \Delta t)(\gamma \Delta t)+\sum_{j=0}^{J} p_{(n+1) 10 j}(1-\lambda \Delta t)(\gamma \Delta t)+p_{(n+1) 110}(1-\lambda \Delta t)(\gamma \Delta t)
\end{gathered}
$$

When $n=2$ :

$$
\begin{aligned}
& p_{n 002} \cong\left(p_{(n-1) 001}+p_{(n-1) 101}\right)(\lambda \Delta t) \\
& p_{n 00 j} \cong p_{(n-1) 10(j-1)}(\lambda \Delta t), \quad \text { for } 3 \leq j \leq J-1 \\
& p_{n 00 J} \cong\left(p_{(n-1) 10(J-1)}+p_{(n-1) 10 J}\right)(\lambda \Delta t)
\end{aligned}
$$

For $n \geq 2$ :

$$
\begin{gathered}
p_{n 10 j} \cong\left(p_{n 00(j-1)}+p_{n 10(j-1)}\right)(1-\lambda \Delta t)(1-\gamma \Delta t)\left(1-\mu_{j} \Delta t\right), \quad \text { for } 2 \leq j \leq J-1 \\
p_{n 10 J} \cong\left(p_{n 00(J-1)}+p_{n 00 J}+p_{n 10(J-1)}+p_{n 10 J}\right)(1-\lambda \Delta t)(1-\gamma \Delta t)\left(1-\mu_{J} \Delta t\right)
\end{gathered}
$$

For $n \geq 3$ :

$$
\begin{aligned}
& p_{n 00 j} \cong\left(p_{(n-1) 00(j-1)}+p_{(n-1) 10(j-1)}\right)(\lambda \Delta t), \quad \text { for } 2 \leq j \leq J-1 \\
& p_{n 00 J} \cong\left(p_{(n-1) 00(J-1)}+p_{(n-1) 00 J}+p_{(n-1) 10(J-1)}+p_{(n-1) 10 J}\right)(\lambda \Delta t)
\end{aligned}
$$

\section{Stationary queue length distribution}

To solve the set of equations derived in Section 3, the following notations are first introduced:

$P_{\sim n}^{*} \quad: \quad$ Column vector containing all the stationary probabilities of size $n$.

$Q_{n, m} \quad$ : Coefficient matrix that relates $P_{\sim}^{*}$ to $P_{\sim}^{*}$.

When $n=1$, Equations (8) to (14) can be represented in the matrix form as follows:

$$
P_{\sim 1}^{*}=\underset{\sim}{Q_{1,0}} P_{\sim 0}^{*}+\underset{\sim}{Q_{1,1}} P_{\sim 1}^{*}+{\underset{\sim}{1,2}}_{\sim 2} P^{*}
$$

By simple matrix operations, we can solve for ${\underset{\sim 1}{P}}^{*}$ in $(22)$ in terms of ${\underset{\sim}{0}}_{0}^{*}$ and ${\underset{\sim}{2}}_{2}^{*}$. The system of equations in (22) can then be simplified to:

$$
P_{\sim 1}^{*}=Q_{\sim, 0}^{s} P_{\sim 0}^{*}+Q_{\sim 1,2}^{s} P_{\sim 2}^{*}
$$

where $\underset{\sim}{Q_{n, m}^{s}}$ is the coefficient matrix that relates $\underset{\sim m}{P_{m}^{*}}$ to $\underset{\sim n}{\underset{P}{*}}$ which is obtained after performing some matrix operations.

When $n=2$, Equations (11) to (19) can be written in the following form:

$$
P_{\sim 2}^{*}={\underset{\sim}{2,1}}_{\sim 1} P^{*}+{\underset{\sim}{2,2}}_{\sim 2} P^{*}+{\underset{\sim}{2,3}}_{\sim 3} P^{*}
$$

By substituting $P_{\sim 1}^{*}$ in (23) into (24) and solve for $P_{\sim 2}^{*}$ in terms of $P_{\sim 0}^{*}$ and $P_{\sim}^{*}$, we can get the following system of equations in the matrix form: 


$$
\underset{\sim 2}{P_{2}^{*}}={\underset{\sim}{2,0}}_{\sim}^{s} \underset{\sim}{P_{0}^{*}}+{\underset{\sim}{\sim}, 3}_{\sim 3}^{s} P^{*}
$$

In general, for $n \geq 3$, we can form the following matrix from Equations (11) to (14) and (18) to (21):

$$
P_{\sim n}^{*}=Q_{\sim, n-1} P_{\sim n-1}^{*}+Q_{n, n} P_{\sim n}^{*}+Q_{n, n+1} P_{\sim n+1}^{*}
$$

The same computation in getting (25) will be performed iteratively to obtain the following matrix equation for $n \geq 3$ :

$$
P_{\sim}^{*}=\underset{\sim}{Q_{n, 0}^{s}} \underset{\sim 0}{*}+{\underset{\sim}{s, n+1}}_{\sim n+1}^{s}
$$

Let $n=N$ be a number that is large enough such that we can set all $P_{(N+1) i r j}$ in (27) to be zero and obtain:

$$
\underset{\sim N}{P_{N}^{*}}=\underset{\sim}{Q_{N, 0}^{f}} \underset{\sim}{P_{0}^{*}}
$$

where ${\underset{\sim}{n, 0}}_{f}^{f}$ is another coefficient matrix that relates $P_{\sim}^{*}$ to $P_{\sim}^{*}$. Substituting (28) into (27) for $n=N-1$ yields the expression:

$$
P_{\sim N-1}^{*}=Q_{\sim-1,0}^{f} P_{0}^{*}
$$

Similarly, for $n=N-2, N-1, \ldots, 1$, by using the iterative procedure, we will obtain:

$$
{\underset{\sim}{P}}_{n}^{*}={\underset{\sim}{n, 0}}_{\sim}^{f}{\underset{\sim}{*}}^{*}
$$

When $n=0$, the following equation can be formed from (6) and (7):

$$
P_{\sim 0}^{*}=Q_{0,0}^{s} P_{\sim 0}^{*}+Q_{\sim, 1}^{s} P_{\sim}^{*}
$$

Let $n=1$ for Equation (30) and by substituting into (31), we get:

$$
P_{\sim 0}^{*}=\underset{\sim}{Q_{0,0}^{f}} P_{\sim}^{*}
$$

Since $\sum_{n=0}^{N} \sum_{i} \sum_{r} \sum_{j} p_{n i j} \cong 1$, the sum of the right hand side (RHS) of (30) for $n=1,2, \ldots, N$ and RHS of (32) will yield an equation of the form:

$$
\sum_{n=0}^{N} \sum_{i} \sum_{r} \sum_{j} c_{n i j} p_{n i j} \cong 1
$$

where $c_{n i r j}$ are constants.

An inspection on the system of equations in (32) reveals that there is one linearly dependent equation. Hence, by substituting one of the equations in (32) with (33), we can then solve for $p_{01 r 0}$ for $r=0,1$. After computing the values of $p_{01 r 0}$, we can easily find the numerical values for all the $p_{n i r j}$ for $n=1,2, \ldots, N$ from Equation (30). The stationary probabilities that there are $n$ positive customers in the queue are then found by

$$
p_{n}=\sum_{i} \sum_{r} \sum_{j} p_{n i r j}
$$

\section{Mean queue length}

When all the values of $p_{n}$ are obtained, the mean queue length can be computed using the following function where $L_{q}$ is the number of positive customer in the queue.

$$
E\left(L_{q}\right) \cong \sum_{n=0}^{\infty} n p_{n}
$$
by

On the other side, from Pollaczek-Khinchine formula, the mean queue length is given 


$$
E(L)=\rho+\frac{\lambda^{2} E\left(S^{2}\right)}{2(1-\rho)}
$$

where $L, S$ and $\rho$ are the queue length random variable, service time random variable and the utilization factor of the queue respectively.

In Harrison and Pitel [9], the modified service time random variable $S_{n e w}$ with pdf $b(t)$ has been found as follows:

$$
b(t)=f(t) e^{-\gamma t}+\gamma(1-F(t)) e^{-\gamma t}
$$

and its corresponding Laplace transform, $\mathscr{L}\{b(t)\}(s)$ is derived as

$$
\mathscr{L}\{b(t)\}(s)=\mathscr{L}\{f(t)\}(s+\gamma)+\frac{\gamma}{s+\gamma}-\gamma \mathscr{L}\{F(t)\}(s+\gamma)
$$

From (38), we can find the utilization factor $\rho_{\text {new }}$ and the second moment of the modified service time distribution $E\left(S_{\text {new }}^{2}\right)$ :

$$
\rho_{\text {new }}=-\lambda \mathscr{L}^{\prime}\{b(t)\}(0) \text {, and } E\left(S_{\text {new }}^{2}\right)=\mathscr{L}^{\prime \prime}\{b(t)\}(0)
$$

where $-\mathscr{L}^{\prime}\{b(t)\}(0)$ and $\mathscr{L}^{\prime \prime}\{b(t)\}(0)$ are the first and second moment of the random variable $S_{\text {new. }}$.

Substituting (39) into (36), the mean queue length of the M/G/1 queue with negative arrivals and $\mathrm{RCH}$ killing strategy can be found by the expression:

$$
E\left(L_{q}\right)=\rho_{\text {new }}+\frac{\lambda^{2} E\left(S_{\text {new }}^{2}\right)}{2\left(1-\rho_{\text {new }}\right)}
$$

\section{Numerical examples}

We first present in tables 1 and 2 the mean queue length of the system and compare the numerical results to those obtained by Equation (40). In table 1, service time for the positive customer is assumed to have exponential distribution with rate $\mu$. Then in table 2 , Gamma distributed service time is considered with shape parameter $\kappa$ and scale parameter $\beta$. Simulation is also carried out to verify the results.

Table 1. Comparison of mean queue length when $f(t)$ follows exponential distribution.

$$
\left[\mu_{k}=\mu \text { for } k=1,2,, \ldots, J\right] \text {. }
$$

\begin{tabular}{|c|c|c|c|c|c|}
\hline \multirow{2}{*}{$1 / \lambda$} & $1 / \gamma$ & $1 / \mu$ & \multicolumn{3}{|c|}{ Mean queue length } \\
\cline { 5 - 6 } & & & Alternative numerical method & Equation (40) & Simulation \\
\hline 2.5 & 3 & 3.5 & 1.826087 & 1.826087 & 1.828284 \\
\hline 0.5 & 0.4 & 0.9 & 1.241379 & 1.241379 & 1.241820 \\
\hline 2.5 & 5.0 & 4.0 & 8.000000 & 8.000000 & 8.022338 \\
\hline 0.4 & 0.2 & 0.5 & 0.555556 & 0.555556 & 0.555835 \\
\hline
\end{tabular}

Table 2. Comparison of mean queue length when $f(t)$ follows gamma distribution.

\begin{tabular}{|c|c|c|c|c|c|}
\hline \multirow{2}{*}{$1 / \lambda$} & \multirow{2}{*}{$1 / \gamma$} & \multirow{2}{*}{$(\kappa, \beta)$} & \multicolumn{3}{|c|}{ Mean queue length } \\
\cline { 4 - 6 } & & & Alternative numerical method & Equation $(40)$ & Simulation \\
\hline 2.0 & 3.0 & $(3,0.2)$ & 0.329861 & 0.329861 & 0.329928 \\
\hline 0.5 & 0.4 & $(2,1.1)$ & 2.797456 & 2.797459 & 2.800176 \\
\hline 1.6 & 2.0 & $(2,0.5)$ & 0.745455 & 0.745455 & 0.745017 \\
\hline 0.8 & 2.0 & $(2,0.5)$ & 7.397671 & 7.400000 & 7.4769897 \\
\hline
\end{tabular}

Tables 1 and 2 show that the numerical results obtained by the alternative method is close to those computed from expression (40). The results have been further confirmed by comparison to those obtained from the simulation procedure. 
In table 3, numerical examples for stationary queue length distributions will be shown in which the service time has a Gamma distribution with fractional values of shape parameter. Steady-state distribution of queue length with such service and/ or interarrival time distributions may not be easily computed by most of the existing analytical methods.

Table 3. Stationary queue length distribution computed using the alternative numerical method and those obtained from the simulation procedure.

\begin{tabular}{|c|c|c|c|c|}
\hline \multirow{2}{*}{$\begin{array}{c}\text { Queue } \\
\text { length } \\
n\end{array}$} & \multicolumn{2}{|c|}{$\begin{array}{c}p_{n} \text { with parameters: } \\
{\left[\begin{array}{l}1 / \lambda=1 / 1.5,1 / \gamma=0.5, \\
(\kappa, \beta)=(1.8,0.3)\end{array}\right]}\end{array}$} & \multicolumn{2}{|c|}{$\begin{array}{c}\text { with parameters: } \\
{\left[\begin{array}{c}1 / \lambda=1.25,1 / \gamma=1 \\
(\kappa, \beta)=(2.3,0.6)\end{array}\right]}\end{array}$} \\
\hline & Alternative numerical method & Simulation & $\begin{array}{c}\text { Alternative numerical } \\
\text { method }\end{array}$ & Simulation \\
\hline 0 & 0.703438 & 0.703268 & 0.174062 & 0.173678 \\
\hline 1 & 0.220301 & 0.220422 & 0.159205 & 0.158918 \\
\hline 2 & 0.057618 & 0.057641 & 0.132878 & 0.132871 \\
\hline 3 & 0.014182 & 0.014194 & 0.107599 & 0.107659 \\
\hline 4 & 0.0034 & 0.003410 & 0.086238 & 0.086251 \\
\hline 5 & 0.000808 & 0.000812 & 0.068877 & 0.068847 \\
\hline 6 & 0.000191 & 0.000193 & 0.054947 & 0.054880 \\
\hline 7 & 0.000045 & 0.000046 & 0.043818 & 0.043846 \\
\hline 8 & 0.000011 & 0.000011 & 0.034939 & 0.035040 \\
\hline 9 & 0.000003 & 0.000004 & 0.027857 & 0.027927 \\
\hline 10 & 0.000001 & 0.000002 & 0.022211 & 0.022264 \\
\hline & $\cdots$ & $\ldots$ & & \\
\hline 50 & 0 & 0 & 0.000003 & 0.000028 \\
\hline
\end{tabular}

From table 3, it can be seen that numerical results computed using the alternative method are close to that obtained by the simulation procedure.

\section{Conclusion}

The alternative method discussed in this paper has been successfully applied to find the stationary queue length distribution for queueing system with negative arrivals. The service time distributions are assumed to have constant asymptotic rates when time $t$ tends to infinity. To find the stationary queue length distribution, the alternative method does not involve derivation of pgf which requires Laplace transform on the pdf and cumulative distribution function of the modified service time random variables which may have a complex form when the distribution is general. Simple iterative procedure is used to solve for the stationary probabilities derived in this paper. The drawback of the alternative method is that we may encounter dimensionality problem when $J$ is large.

\section{Acknowledgments}

This work was supported by MOHE FRGS under vote account number 4471/K01.

\section{References}

1. Gelenbe E 1989 Random neural networks with negative and positive signals and product form solution Neural Comput. 1 pp 502-510

2. Gelenbe E 1991 Product-form queueing networks with negative and positive customers J. Appl. Prob. 28 pp 656-663 
3. Gelenbe E 1994 G-networks: a unifying model for neural and queueing networks Ann. Oper. Res. 48 pp 433-461

4. Artalejo J R 2000 G-networks: a versatile approach for work removal in queueing networks Eur. J. Oper. Res. 126 pp 233-249

5. Do T V 2011 An initiative for a classified bibliography on G-networks Performance Eval. 68 (4) pp 385-394

6. Jain G and Sigman K 1996 A Pollaczek-Khintchine formula for M/G/1 queues with disasters J. Appl. Prob 33 (4) pp 1191-1200

7. Boucherie R J and Boxma O J 1996 The workload in the M/G/1 queue with work removal Probability in the Engineering and Informational Sciences 10 (2) pp 261-277

8. Harrison P G and Pitel E 1993 Sojourn Times in Single-server queues with negative customers J. Appl. Prob. 30 pp 943-963

9. Harrison P G and Pitel E 1996 The M/G/1 queue with negative customers Adv. Appl. Prob. 28 pp 540-566

10. Koh S K 2013 Maintenance of deteriorating non-exponential single server queue $\mathrm{PhD}$ Thesis (Malaysia: University of Malaya) 